\title{
Gender Differences in Preventing the Spread of Coronavirus
}

\author{
Irmak Olcaysoy Okten ${ }^{1}$ \\ Anton Gollwitzer ${ }^{2}$
}

\author{
Gabriele Oettingen ${ }^{1}$ \\ ${ }^{1}$ Department of Psychology, New York University \\ ${ }^{2}$ Department of Psychology, Yale University
}

Word Count: 6679 (including footnotes), 4473 (not including footnotes)
*********THE FOLLOWING CONTENT HAS NOT BEEN PEER REVIEWED******** IF YOU REFERENCE THIS ARTICLE IN ANY FORM PLEASE INDICATE
THAT IT HAS NOT BEEN PEER REVIEWED- THANK YOU

\begin{abstract}
Author Note
Authors have no conflicts of interest to disclose.

Correspondence concerning this article should be addressed to Irmak Olcaysoy Okten,

6 Washington Place, New York, NY 10003. Email: irmakolcaysoy@gmail.com
\end{abstract}




\begin{abstract}
Social distancing and hygiene practices are key to preventing the spread of Coronavirus. However, people vary in the degree to which they follow these practices. Consistent with previous findings that women adhere more to preventative health practices, in Study 1, women reported engaging in more preventative practices regarding COVID-19 (e.g., social distancing, hygiene) than men. In Study 2, across three different Northeast U.S. locations, we observed a greater percentage of women wearing masks in public than men. In Study 3, U.S. counties with a greater percentage of women exhibited greater social distancing as tracked by the geo-location of $\sim 15$ million GPS smart-phones per day. These findings suggest that preventive health messages should be tuned especially towards men.
\end{abstract}

Keywords: gender, COVID-19, Coronavirus, preventive practices, social distancing, health 


\section{Gender Differences in Preventing the Spread of Coronavirus}

The guidelines on how to prevent the spread of COVID-19 have been straightforward. ${ }^{1}$ Medical experts have unanimously emphasized the importance of social distancing ${ }^{\mathrm{i}}$ (avoiding physical contact with others) and personal hygiene to minimize the spread of the virus. Yet, despite these straightforward guidelines, individuals and communities vary in their health behaviors. ${ }^{2,3,4}$ While some carefully followed shelter-in-place orders, others enjoyed packed beaches or pub crawls. ${ }^{5,6}$ Elucidating individual and group differences underlying these behaviors should inform policy makers' understanding of how to motivate people (and whom to motivate) to engage in preventative measures during viral pandemics.

Here we examine whether gender helps explain variance in people's and group's responses to COVID-19. Women may respond more effectively than men. For one, women endorse preventive health practices more so in their daily lives; they visit and comply with doctors and participate in preventive health services more than men. ${ }^{7}$ Additionally, women pay more attention than men to their own and others' health-related needs, and show greater empathic reactivity in response to others' pain. ${ }^{8,9,10,11,12,13,14}$ Women are also more likely to avoid taking risks in terms of their health, as well as in terms of other behaviors and decisions. ${ }^{15}$, ${ }^{16}$ Building on this literature, the current COVID-19 pandemic provides a unique opportunity to test whether women are more efficacious in terms of health behaviors on a grand scale. Specifically, we predicted that women are more likely to comply with preventive practices (e.g., social distancing, maintaining hygiene), listen to experts when deciding to what extent to social distance, and exhibit greater alarm and anxiety in response to COVID-19.

\footnotetext{
i We use the term "social distancing" to refer to physical, in-person contact with others rather than "physical distancing" in the present paper because of the greater frequency of usage of the former term than the latter term (approximately 964 million vs. 21 million results on Google Search, respectively, on July 4, 2020).
} 


\section{Study 1}

\section{Method}

\section{Participants}

We recruited 800 participants from the U.S. via Prolific (see for participant recruitment details osf.io/x8uvc). ${ }^{\text {ii }}$ Participants completed a five-minute survey that was programmed on Qualtrics for $\$ 0.55$ on April 8, 2020. Thirty participants were excluded from the analyses; 10 for failing an attention check, and 20 because their gender was nonbinary $(N=770 ; 442$ Female; $M_{\text {age }}=30.7$, Median $\left._{\text {age }}=27.7, S D_{\text {age }}=11.04\right) .{ }^{\text {iii }}$ The distribution of participant ethnicity was as follows: $61.9 \%$ White, 7.4\% Black or African American, 13.7\% Asian, 9.4\% Hispanic, 5.4\% Mixed, 0.7\% American Indian, 0.1\% Native Hawaiian, 1.7\% Other. . $^{\text {iv }}$

\section{Measures}

Preventative Practices. Five questions assessed preventative COVID-19 practices: (1) participants' reported number of days with in-person contact in the past week (0-7 days), (2) the number of days with in-person contact with friends and family in the past week (0-7 days), (3) frequency of hand-washing, (4) staying at home (other than shopping for groceries), and (5) maintaining six feet distance from others (https://osf.io/bkqj7 for verbatim materials; e.g.; "I have been washing my hands more often," 1: Strongly Disagree, 7: Strongly Agree). Importantly, similar self-report items have been linked to actual social distancing behavior in terms of decreased movement (as measured by step-counters and GPS tracking data; Gollwitzer, Martel, Marshall, Höhs, \& Bargh, 2020). ${ }^{17}$

\footnotetext{
ii An a-priori power analysis suggested a sample size of 788 to estimate a small effect size $(d=.2)$ with a power of .80 and an alpha of .05 . We recruited 800 participants to account for participant exclusion.

iii Men and women in the sample did not differ in their level of education, $\chi^{2}(6)=9.72, p=.14$.

iv The distribution of White individuals in our sample matched the proportion in the U.S. population (U.S. population in 2018: 60.4\%). However, compared to U.S. population, our sample was younger (Median = 38.2; U. S. Census, 2018), and included more Asian (U.S. population: 5.9\%) and a lower number of Black (U.S. population in 2018: 13.4\%) and Hispanic individuals (U.S. population in 2018: 18.3\%).
} 
Source of Information for Social Distancing. We assessed individuals' reported reliance on external sources (medical experts, the president, religious leaders, their governor, national media, social media, other countries' experiences, their family, their friends, their neighbors) and internal sources (i.e., information that is driven from one's own experiences and feelings rather than other entities; one's responsibility for others, one's responsibility for themselves, one's health history, one's anxiety) when deciding to what extent to socially distance: "How are the following factors influencing to what extent you are socially distancing yourself from others? (1: Not at all, 7: Very much) (Table 1).

Psychological Experience. Participants reported their anxiety ("Thinking about Covid19 makes me feel extremely anxious"; 1: Strongly disagree, 7: Strongly agree), preoccupation ("How much preoccupied are you by the current Coronavirus pandemic?"; 1: Not at all, 7: Extremely), and uncertainty regarding Covid-19 ( "How much uncertainty do you experience in your daily life as a result of the current Coronavirus pandemic?”; 1: Not at all, 7: Extremely).

Other Factors. Participants reported their daily frequency of checking COVID-19 news (open-ended) and how knowledgeable they feel about COVID-19 (1: Not at all, 7: Extremely). They also reported whether they belong to a vulnerable population for contracting COVID-19, whether they know anyone who contracted COVID-19, ${ }^{\mathrm{v}}$ their expectancy of contracting COVID19 (1: Not at all likely, 7: Very likely), how important it is to them to not contract COVID-19 (1: Not at all important, 7: Very important), and how much their daily routines changed due to COVID-19 (1: Not at all, 7: Extremely). All the questionnaires and scales were presented in randomized order. Finally, we assessed social desirability in responding, ${ }^{18}$ number of on-site

\footnotetext{
${ }^{v}$ A short COVID-19 quiz with three items was also included in the survey. However, because of the low statistical reliability of this quiz, we do not discuss the quiz here.
} 
workdays in the past week (0-7 days), and demographics, including political orientation (1: Very

\author{
Conservative, 7: Very Liberal)."
}

\title{
Results
}

\section{Preventive Practices}

Females reported greater preventive practices on four of the five items (i.e., maintaining

six feet distance and hygiene, staying at home, and lower in-person contact with others; Table 1).

The only item without a gender difference was the frequency of in-person contact with people

other than family/friends, though the means were in the predicted direction. ${ }^{\mathrm{vi}, \text { vii, viii, ix }}$

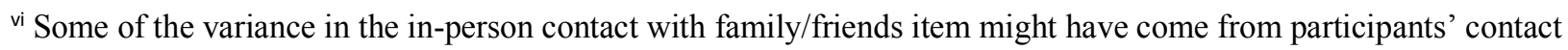
with immediate family who are quarantining with the participant. However, the effect of gender on this item echoed that of the other social distancing items, indicating that though some variability on this item may come from inperson contact with one's immediate family living in the same household, part of the variance is likely captured by in-person contact with extended family and friends not living in the same household.

vii Exploratory analyses showed gender differences on this item when the political ideology of participants was considered. Gender interacted with political ideology in predicting face-to-face contact with others, $b=-.21, t(762)$ $=-2.06, p=.04$. Conservatism among males (but not females) significantly predicted greater face-to-face contact with others. The gender difference among conservative participants $(+1 S D)$ was significant, $b=.45, t(762)=2.14, p$ $=.033$, although it was not significant among liberal participants $(-1 S D), b=-.18, t(762)=-.811, p=.418$.

viii The tendency to go outside during a pandemic might create different levels of risk depending on the density of population where one is located. Therefore, in an additional analysis examining gender differences on the staying at home item, we added participants' location at the time (categorized as urban vs. not urban based on the population density information extracted from the zip codes as in: https://github.com/ibm-watson-data-lab/opendata/tree/master/urbanity). Specifically, we added Location (urban vs. not urban) and Gender in a factorial ANOVA to predict the tendency to stay at home. This analysis revealed a non-significant interaction between Gender and Location, $F(1,755)=.903, p=.342, \eta_{\mathrm{p}}^{2}=.001$, in predicting staying at home.

${ }^{i x}$ We also tested age, ethnicity, and education as potential moderators of the gender differences observed. The only analysis where the interaction between age and gender was significant was for in-person contact with others, $F(1$, $701)=4.17, p=042, \eta_{\mathrm{p}}{ }^{2}=.01$, suggesting a greater gender difference (women's less frequent contact with others) among older participants. Age did not moderate gender differences in any other measures, $p \mathrm{~s}>.1$. The only finding that was affected by participant ethnicity was the tendency to stay at home other than shopping, $F(3,701)=3.54, p$ $=.014, \eta_{\mathrm{p}}{ }^{2}=.015$. Although the observed gender difference was significant among White and Black participants $(p \mathrm{~s}$ $<.03$ ), the difference did not reach statistical significance for Asian and Hispanic participants. Ethnicity did not interact with gender significantly in any of the other measures, $p \mathrm{~s}>.1$. The level of education (analyzed in two categories; 1. Bachelor's degree or higher, and 2. lower than Bachelor's degree) did not significantly interact with gender in predicting any of the reported effects except for hand washing frequency, $F(1,766)=5.14, p=.024, \eta_{\mathrm{p}}{ }^{2}$ $=.01$. Only within that measure, individuals with a lower level of education showed greater gender differences (women washing their hands more frequently than men) than individuals with a higher level of education. An exploratory analysis on the interaction of gender and education in predicting in-person contact with others also revealed a significant interaction, $F(1,763)=7.05, p=.008, \eta_{\mathrm{p}}{ }^{2}=.01$, suggesting a gender difference among those with a higher level of education; men were more likely to report in-person contact with others than women within that group, $F(1,763)=8.20, p=.004, \eta_{\mathrm{p}}^{2}=.011$. The gender difference was not significant among men and women with a lower level of education, $F(1,763)=.665, p=.415, \eta_{\mathrm{p}}{ }^{2}=.001$.
} 
Table 1. Summary of Study 1 Findings

\begin{tabular}{|c|c|c|c|c|c|c|c|c|}
\hline & $\begin{array}{c}\text { Women } \\
(N=442)\end{array}$ & $\begin{array}{c}\text { Men } \\
(N=328)\end{array}$ & \multirow{2}{*}{$t$} & \multirow{2}{*}{$d f$} & \multirow{2}{*}{$p$} & \multirow{2}{*}{ Lower } & \multirow{2}{*}{ Upper } & \multirow{2}{*}{$\begin{array}{c}\text { Cohen's } \\
d\end{array}$} \\
\hline & $\begin{array}{l}\text { Mean } \\
(S D)\end{array}$ & $\begin{array}{l}\text { Mean } \\
(S D)\end{array}$ & & & & & & \\
\hline $\begin{array}{l}\text { Preventive Practices } \\
\text { In person contact with } \\
\text { family or friends } \\
\text { (\# days/a week) }\end{array}$ & $4.18(2.97)$ & $4.72(2.86)$ & 2.54 & 719.29 & .011 & .12 & .96 & 0.19 \\
\hline $\begin{array}{l}\text { In person contact with } \\
\text { others (\# days/a week) }\end{array}$ & $1.61(2.07)$ & $1.81(2.08)$ & 1.31 & 765 & .191 & -.10 & .49 & 0.09 \\
\hline Handwashing & $6.37(1.07)$ & $6.17(1.25)$ & -2.33 & 768 & .020 & -.36 & -.03 & -0.17 \\
\hline $\begin{array}{l}\text { Staying at home (other } \\
\text { than shopping) }\end{array}$ & $5.83(1.65)$ & $5.51(1.83)$ & -2.49 & 662.52 & .013 & -.57 & -.07 & -0.19 \\
\hline $\begin{array}{l}\text { Attention to maintaining } \\
\text { six feet distance }\end{array}$ & $6.29(1.14)$ & $6.03(1.20)$ & -2.99 & 768 & .003 & -.42 & -.09 & -0.22 \\
\hline
\end{tabular}

\section{Source of Information for Social Distancing}

External Sources for Social Distancing

The president

Religious leaders

Your governor

Medical experts

National media

Social media

Other countries

Your family

Your friends

Your neighbors
$2.87(2.09)$

$2.03(1.73)$

$5.03(1.95)$

$6.23(1.24)$

$4.75(1.78)$

$3.93(2.06)$

$5.51(1.75)$

4.62(2.01)

$3.74(1.97)$

2.51(1.84)
$2.91(1.93) \quad .289$

$1.98(1.65) \quad-.367$

$4.48(1.87) \quad-3.88$

$5.98(1.36) \quad-2.64$

$4.29(1.72) \quad-3.62$

$3.51(1.85) \quad-2.99$

$5.15(1.69) \quad-2.90$

$4.68(1.82) \quad .438$

$3.76(1.88) \quad 1.37$

$2.34(1.71) \quad-.551$
$732.65 \quad .775$

768

768

768

768

740.84

768

737.34

768

730.56 $\begin{array}{ll}-.24 & .33\end{array}$

$\begin{array}{lll}.714 & -.29 & .20\end{array}$

$\begin{array}{lll}<.001 & -.82 & -.27\end{array}$

$\begin{array}{lll}.009 & -.43 & -.06\end{array}$

$\begin{array}{lll}<.001 & -71 & -.21\end{array}$

$\begin{array}{ll}-.70 & -.14\end{array}$

$\begin{array}{ll}-.61 & -.12\end{array}$

$\begin{array}{ll}-.21 & .33\end{array}$

$\begin{array}{ll}-.26 & .29\end{array}$

$-.32 \quad .18$
0.02

$-0.03$

$-0.28$

$-0.19$

$-0.26$

$-0.22$

$-0.21$

0.03

0.10

$-0.04$

Internal Sources for Social Distancing

Your health history

Your anxiety

Your feeling of

responsibility for others

Your feeling of

responsibility for yourself

Psychological Experience

Feeling extremely

anxious

Feeling preoccupied

Feeling uncertain
$4.92(1.92)$

$3.52(1.99)$

$4.04(1.90)$

$6.10(1.34)$

$5.78(1.32)$

$6.06(1.34)$
-3.69
-7.32

744.14

$<.001$

$<.001$

$-.87$

$-1.16$

768

.001

$-.51$

680.96

$<.001$

$-.56$

$-.26$

$-.61$

$-0.27$

$-0.46$

$-0.24$

$-0.28$

\section{Other Factors}

$\begin{array}{llllllll}4.94(1.65) & 4.09(1.67) & -7.06 & 768 & <.001 & -1.09 & -.61 & -0.51 \\ & & & & & & & \\ 4.71(1.50) & 4.41(1.55) & -2.72 & 768 & .007 & -.52 & -.08 & -0.20 \\ 4.88(1.56) & 4.61(1.57) & -2.42 & 768 & .016 & -.50 & -.05 & -0.17\end{array}$




\begin{tabular}{|c|c|c|c|c|c|c|c|c|}
\hline Subjective knowledge & $5.22(1.09)$ & $5.09(1.07)$ & -1.7 & 768 & .089 & -.29 & .02 & -0.12 \\
\hline $\begin{array}{l}\text { Frequency of checking } \\
\text { news }\end{array}$ & $3.88(4.43)$ & $3.82(4.04)$ & -.218 & 756 & .828 & -.68 & .55 & -0.02 \\
\hline Social desirability & $.43(.23)$ & $.41(.23)$ & -.940 & 767 & .348 & -.05 & .02 & -0.07 \\
\hline $\begin{array}{l}\text { Number of on-site } \\
\text { workdays (/a week) }\end{array}$ & $.75(1.70)$ & $.98(1.89)$ & 1.71 & 657.17 & .088 & -.03 & .49 & 0.13 \\
\hline Change in routines & $5.33(1.67)$ & $5.27(1.63)$ & -.477 & 768 & .634 & -29 & .18 & -0.03 \\
\hline $\begin{array}{l}\text { Expectancy of getting the } \\
\text { virus }\end{array}$ & $3.72(1.48)$ & $3.58(1.45)$ & -1.32 & 768 & .188 & -.35 & .07 & -0.09 \\
\hline $\begin{array}{l}\text { Importance of not getting } \\
\text { the virus }\end{array}$ & $5.88(1.41)$ & $5.68(1.51)$ & -1.89 & 768 & .060 & -.41 & .01 & -0.14 \\
\hline
\end{tabular}

Note. We report adjusted $t$, df and $p$ values in comparisons where the equality of variance assumption was not met.

\section{Sources of Information for Social Distancing}

Females reported relying on information from data-driven sources (medical experts, their governor, other countries' experiences, media) more so than males when deciding to what extent to social distance. Females and males, however, were similarly likely to rely on less dataoriented external sources, such as the president, religious leaders, and familiar others. Additionally, females reported being influenced by all four internal sources (health history, anxiety, feeling responsible for others, feeling responsible for oneself) more so than males.

\section{Psychological Experience}

Females reported experiencing greater negative emotions (anxiety, preoccupation, uncertainty) regarding COVID-19 than males.

\section{Other Factors}

Males and females did not differ in how knowledgeable they felt about COVID-19 and in their frequency of checking COVID-19 news. ${ }^{\mathrm{x}}$ They also did not differ in having contracted

\footnotetext{
x 12 participants who did not report their frequency of checking news were excluded from the analysis.
} 
COVID, or knowing someone infected by the virus, $\chi^{2}(6)=2.77, p=.84 .{ }^{\text {xixii }}$ They did not differ in their expectancy of contracting the virus, perceived importance of not becoming infected, social desirability, and change in daily routines; thus, these variables are unlikely to account for the observed findings. Importantly, although the difference in the number of on-site workdays approached significance, controlling for this factor did not change the reported gender differences.

Males in our sample were more conservative than females, $t(767)=4.44, p<.001$. When controlling for political conservatism many of the observed effects reduced in size - the decrease of Cohen's $d$ varied between $43 \%$ and $6 \%$ - though the effects remained largely significant (only one of the effects became nonsignificant: the difference in seeking information from medical experts, $F(1,756)=2.62, p=.11)$. ${ }^{\text {xiii }}$ These results suggest that a latent factor underlying male gender and conservatism may in part explain the observed gender differences. Future research should test whether psychological constructs related to both maleness and conservatism, for instance, high-power, greater assertiveness, and feelings of autonomy and independence 10, 17, 19, ${ }^{20}$ may help explain the observed gender differences.

\section{Study 2}

Though social desirability did not account for the self-report findings of Study 1, it remains possible that gender differences in reporting rather than in actual behavior underlie the

\footnotetext{
${ }^{x i}$ Most participants did not personally know someone who contracted the virus (Females: 75.6\%, Males: 75.9\%). Three males and one female reported having had the virus themselves.

xii A comparison of the distributions of participants (within each gender) in terms of their reported vulnerabilities to COVID-19 revealed some significant gender differences, $\chi^{2}(5)=28.89, p<.001$. Specifically, a chi-square analysis showed that females were more likely to report vulnerabilities due to their health history (Females: 19.2\%, Males: 10.7\%), profession (Females: $7.9 \%$, Males: $4 \%$ ) and other reasons (Females: 5.4\%, Males: $2.1 \%$ ) compared to males. Controlling for reported vulnerabilities did not affect the observed gender differences.

xiii Exploratory analyses revealed an interaction between gender and political orientation on this item, $b=-.21$, $t(765)=-3.26, p=.001$. Among conservatives, males exhibited a greater reluctance to listen to medical experts than females; the gender difference among conservative participants $(+1 S D)$ was significant, $b=-.44, t(765)=-3.43, p=$ .001 . This link was not significant among liberal participants $(-1 S D), b=.17, t(765)=1.26, p=.210$.
} 
observed findings. To address this concern, in Study 2, we utilized observational methodologies to test whether men and women differ in terms of COVID-19 prevention. Observational methods entail greater ecological validity than self-report measures as they capture real-world behavior. 21, 22, 23, 24 Notably, such methods are also useful for demonstrating the robustness of findings, as these measures are applied in field settings that include numerous uncontrolled factors (i.e., noise). ${ }^{25}$ And furthermore, these methods tend to be easily interpretable and relevant to the people outside of the specific field of psychology. Therefore, in Study 2, we examined whether gender differences exist in terms of public mask-wearing during the COVID-19 pandemic. Based on the results of Study 1, and previous work on gender differences in preventive health behavior, we predicted that women would be more likely to wear masks than men.

\section{Method}

\section{Observation Locations}

We conducted our observations in three different U.S. locations, specifically, New York City, NY (10012), New Haven, CT (06511), and New Brunswick, NJ (08901). Though these locations are all in the northeast of the U.S., they differ on a variety of demographic variables. The three locations varied in terms of annual income per household at the zip-code level (NY [10012]: \$104561, CT [06511]: \$38614, NJ [08901]: \$38413), the distribution of race/ethnicity, (NY [10012]: 75.8\% White, 3.22\% Black, 17.65\% Asian, 0.51\% American Indian or Alaskan Native; 0.15\% Native Hawaiian or other Pacific Islander, 2.66\% Other; CT [06511]: 39.74\% White, 42.63\% Black, 8.23\% Asian, 1.51\% American Indian or Alaskan Native, $0.16 \%$ Native Hawaiian or other Pacific Islander, 6.73\% Other; NJ [08901]: 46.56\% White, 16.77\% Black, 7.99\% Asian, $1.76 \%$ American Indian or Alaskan Native, $0.2 \%$ Native Hawaiian or other Pacific Islander, 26.72\% Other), the median age of the inhabitants (NY [10012]: 34.5, CT 
[06511]: 28.8, NJ [08901]: 23.3) and the number of persons per household (NY [10012]: 1.71, CT [06511]: 2.26, NJ: 3.36 [08901]). ${ }^{\text {xiv }}$ The distribution of male and female inhabitants was similar across the three locations (NY [10012]: 50.85\% Female, CT [06511]: 50.97\% Female, NJ [08901]: 48.79\% Female). All three observation locations involved main streets with paved sidewalks that are convenient for walking.

\section{Participants}

Based on our preliminary observation in these locations, we estimated one pedestrian every 1-2 minutes. As we aimed to complete our observation within two hours, we decided to limit our sample to 100 people in each area and pre-registered this plan (see:

https://aspredicted.org/blind.php? $\mathrm{x}=\mathrm{cf} 3 \mathrm{ea} 8$ ). As pre-registered, each author observed 100 individuals in each location on May 4, 2020 127 females and 173 males were observed in total.

\section{Procedure}

As the observers were self-quarantining in their apartments in three different locations, they picked one street or walked several blocks that were most adjacent to their apartments for observation in each location. Each observer tallied the gender of observed individuals (including individuals on bikes, but not those in cars) and whether they were wearing a mask or not. Wearing a mask meant that individuals had their chin, mouth, and nose covered (whether with cloth or with an actual mask). Individuals who had masks around their neck or in their hands were counted as not wearing a mask.

\section{Results}

\footnotetext{
xiv The demographic data for each location was obtained from here: https://www.cdxtech.com/tools/demographicdata/

${ }^{x v}$ Because of the scarcity of people on the selected street in New Brunswick, NJ, the observer in that location completed the observation on May 5. The timing of observation in each location was as follows: NY: 5 pm - 7 pm; CT: $3.30 \mathrm{pm}-5.30 \mathrm{pm}$; NJ: $3 \mathrm{pm}-8 \mathrm{pm}, 9 \mathrm{am}-12.30 \mathrm{pm}$.
} 
As predicted, a chi-square test of independence revealed that there was a significant association between gender and mask-wearing, with women being more likely to wear masks than men as compared to chance, $\chi^{2}(1)=9.11, p=.003$ (see Table 2). Regarding simple effects, a z-test comparing the proportions conducted on SPSS showed that within women the proportion of those with a mask (55.1\%) was significantly higher than the proportion of those without a mask at the .05 level (44.9\%). In contrast, within men the proportion of those with a mask (37.6\%) was significantly lower than the proportion of those without a mask (62.4\%). Although we did not predict to find gender differences in the number of people in public, we observed a greater proportion of men $(57.7 \%)$ than women $(42.3 \%)$ on the street, $\chi^{2}(1)=7.05, p=.008$. These results align with the finding of Study 1 that women reported a higher tendency to stay at home. ${ }^{\text {xvi }}$

Table 2. Gender * Mask Crosstabulation

\begin{tabular}{|c|c|c|c|c|c|}
\hline & & & \multicolumn{2}{|c|}{ Mask } & \multirow[b]{2}{*}{ Total } \\
\hline & & & No Mask & Mask & \\
\hline \multirow[t]{6}{*}{$\overline{\text { Gender }}$} & Male & Count & $108_{a}$ & $65_{b}$ & 173 \\
\hline & & Expected Count & 95.2 & 77.9 & 173.0 \\
\hline & & $\%$ within Gender & $62.4 \%$ & $37.6 \%$ & $100.0 \%$ \\
\hline & & $\%$ within Mask & $65.5 \%$ & $48.1 \%$ & $57.7 \%$ \\
\hline & & $\%$ of Total & $36.0 \%$ & $21.7 \%$ & $57.7 \%$ \\
\hline & $\overline{\text { Femal }}$ & Count & $57 \mathrm{a}$ & $70_{b}$ & 127 \\
\hline
\end{tabular}

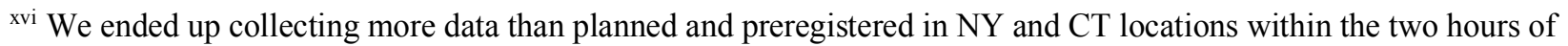
observation. We report the results based on the first 100 observations in each location in accordance with the preregistered plan. Importantly, the results remained the same when we included all collected observations $(\mathrm{N}=777)$ in the analysis, $\chi^{2}(1)=39.58, p<.001$. 


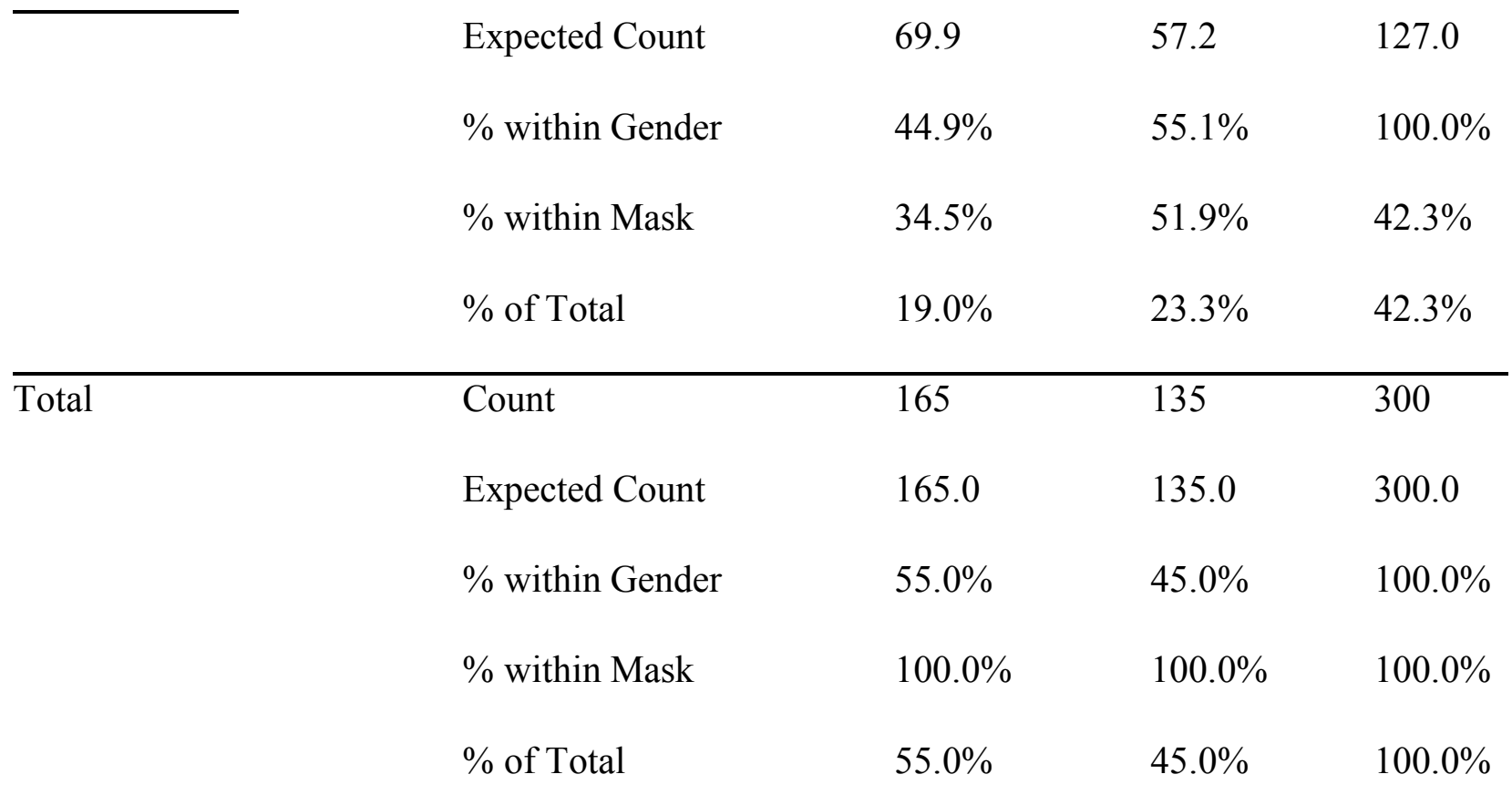

Note. Each subscript letter denotes a subset of Mask categories whose column proportions do not differ significantly from each other at the .05 level.

\section{Study 3}

Building on the self-reported gender-differences in preventive COVID-19 health practices observed in Study 1, in Study 2, we found that women are more likely than men to engage in COVID-19 preventive practices on a behavioral measure. Specifically, we observed that women are more likely to wear masks in public than men (in three different locations in the U.S. Northeast).

Notably, though, the samples of Studies 1 and 2 were not completely representative of the U.S. population. The sample of Study 1 differed from the general population in terms of age, ethnicity, and education level, and the sample we recruited in Study 2 was limited to three specific U.S. locations. Therefore, in Study 3, we tested whether our results extend to social distancing behavior at the U.S. County level. Using the aggregated GPS geo-tracking data of 15 million people around the U.S. per day (tracked via individuals' smart-phone GPS location 
coordinates), we examined whether the gender makeup of approximately 3,000 U.S. Counties predicts the extent to which people in these Counties practiced social distancing during the start of the COVID-19 pandemic (between March 9 and May 29, 2020). Social distancing was measured via (1) overall reduction in movement and (2) reduction in visiting non-essential retail and services as compared to pre-COVID (before March $9^{\text {th }}$ ) as assessed by the GPS tracking data.

\section{Method}

\section{Participants}

The aggregated movement of approximately 15 million people across the U.S. per day between March 9 and May 29, 2020 were shared with the authors by Unacast. ${ }^{26}$ These data are anonymized in that they aggregate GPS coordinates by County. 3,054 Counties were included in the dataset. 29 Counties with 2,000 or less inhabitants were removed from this number for the analyses. 952 additional counties were excluded specifically from the analyses involving visiting non-essential retail and services because of missing data.

\section{Measures}

Social Distancing. Social distancing was assessed in two ways: Decrease in overall movement, and decrease in visiting non-essential retail and services as compared to pre-Covid19 times (before March $9^{\text {th }}$; individually controlled for in each county). ${ }^{\text {xvii }}$ These two behavioral variables - general movement and visiting non-essential retail - were calculated by Unacast (a software company that provides location and map services) using GPS tracking data and then

\footnotetext{
xvii Non-essential retail and services is anything that falls within restaurants, department stores, clothing stores, footwear, discount stores, jewelry, computers \& consumer electronics, gifts, seasonal, books, office supplies, hair, cosmetics and beauty supplies, Gyms + Fitness Facilities, Communications, New/Used Car Dealers, Hotels, Used Products, "Crafts, Toys, and Hobbies", Travel, "Spa, Massage, + Esthetics", Sports + Recreation, Weight Loss, Furnishings, Home + Housewares, Home Improvement +Building Supplies, "Printing, Copying + Publishing", Theatres, Music, Amusement, Furnishing Rentals, Shared Offices + Coworking, Car Wash, Cannabis Retail, Flowers, bars, pubs, cafes, nightclubs, cinemas, casinos.
} 
shared with the authors. These data were anonymized as they were aggregated at the U.S.

County-level. For more details see the SupplementsStudy3.pdf file hosted on our OSF project

page, http://osf.io/bkqj7.

County Gender. Counties' gender breakdowns were provided by https://github.com/JieYingWu/COVID-19_US_County-level_Summaries.

Covariates. Descriptions of the control variables included in our models and the coding of these variables can be found in the SupplementsStudy3.pdf file hosted on our OSF project page, http://osf.io/bkqj7 (Table S1). For a list of the included control variables, see the Results section below.

\section{Results}

We examined whether gender-assessed via the percentage of males versus females in a county_predicted counties' degree of social distancing between March 9 and May 29, 2020. To do so, we conducted a series of three-level mixed-effects models (observations were nested within county and county within state) varying in model specification and saturation. In each of these models, we included random intercepts of county and state and the random slope of gender at the county and state-level (where possible, that is, when doing so did not lead to convergence failures). ${ }^{\text {xiii }}$

First, as base models, we conducted two mixed effects models with reduction in general movement and reduction in visiting nonessential retail as the outcome variables, respectively,

\footnotetext{
xviii We validated the two included behavioral social distancing measures by running mixed-effects models with time (linear and quadratic; orthogonal terms), weekend, median income, stay-at-home order, and COVID cases per capita as fixed effects (see Supplements hosted on OSF here for coding). In line with social distancing policies being instituted around mid-march and being loosened mid/end April, social distancing increased and then decreased over time, $p \mathrm{~s}<.001$ (see Supplements hosted on OSF here; Fig. S1, Fig. S2, Table S2). Furthermore, as expected, social distancing - both in terms of reduced general movement and visiting to non-essential retail - was higher in places with higher per capita rates of infection, was higher on the weekends, was higher in high-income Counties (where people are more likely to be able to work from home), and was higher when stay-at-home policies were in place, $p$ s $<.001$ (Table S2).
} 
and gender distribution ([total \# of males]/[total \# of males + total \# of females] $\left.]^{*} 100\right)(M=$ $50.07, S D=2.26, \min =43.13, \max =73.16)$ as a single fixed effect predictor $(z$-scored $)$. We observed a main effect of gender in that between March 9 and May 29, 2020 counties with a greater proportion of males $(+2 S D)$ reduced their general movement $4.02 \%$ points less, and reduced their visiting of nonessential retail $9.08 \%$ points less, $B_{\text {movement }}=-2.01,95 \% \mathrm{CI}[-2.79$, 1.21], $p<.001$, and $B_{\text {visitation }}=-4.54,95 \% \mathrm{CI}[-5.89,-3.18], p<.001$ (see Base Model in Tables S3 and S4 [see the SupplementsStudy3.pdf file hosted on our OSF page, here]; see Figure 1).

\section{Figure 1}

Percentage Increase in Social Distancing (between March 9 and May 29, 2020) at the County Level as a Function of the Percentage of Male Constituents when Not Accounting for Any Control Variables (Raw Scores).

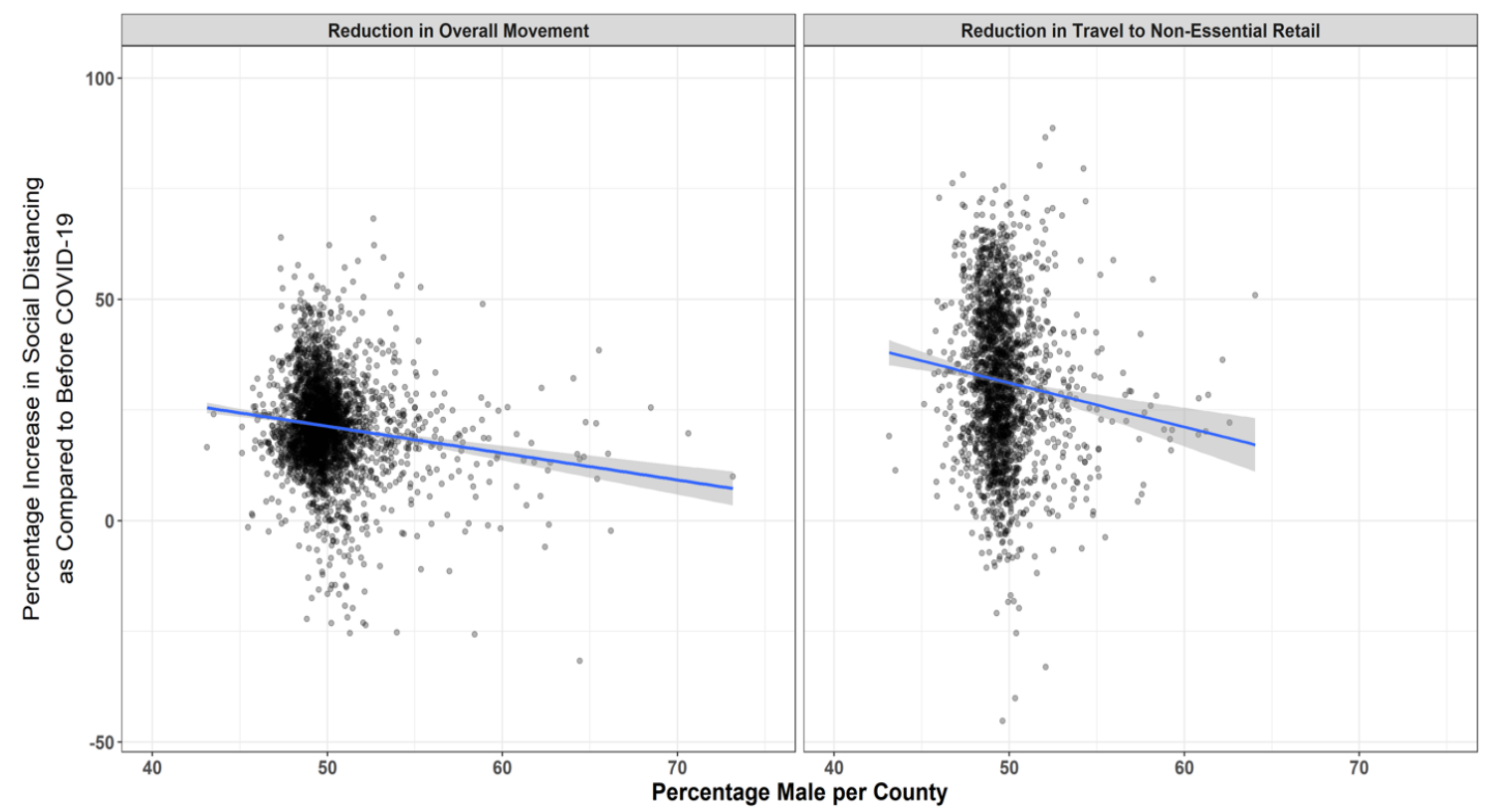

To examine how the observed link between gender distribution and social distancing changed over time (between March 9 and May 29, 2020) and to examine the robustness of this link, we reran these models while adding interaction terms between gender distribution and 
time as well as adding covariates (i.e., potential third-variables). Namely, we ran two further model specifications, a main effects model (including all covariates as fixed effects and the interaction terms between gender and linear time $[z$-scored $]$ and gender and quadratic time as fixed effects [z-scored]), and a fully saturated model (including all covariates, all interaction terms between these covariates and linear time [z-scored], and the interaction term between gender and linear time [ $z$-scored]). The covariates included in these models were: COVID-19 cases per capita (cumulative cases divided by county population; included for each specific day in the included date range; $z$-scored $)$, state policy $(1=$ stay-at-home order not in effect for a specific state on a specific day in the included date range and $0=$ stay-at-home order in effect for a specific state on a specific day in the included date range), weekend $(0=$ Weekday, $1=$ Weekend $),$ median income ( $z$-scored), median age ( $z$-scored), population density (in terms of population per square mile of land area; $z$-scored), religiosity ( $z$-scored; rate of religious adherents per 1000 people), percent employment (z-scored), economic inequality (Gini coefficient; $z$-scored), percentage of adults who only have a high school diploma ( $z$-scored), percentage of adults with a college degree below bachelor's ( $z$-scored), and percentage of adults who have at least a bachelor's degree (z-scored) (see Table S1 in the SupplementsStudy3.pdf file hosted on our OSF project page, http://osf.io/bkqj7, for descriptions and sources).

Importantly, we observed negative interactions between counties' gender distribution and linear time both for the main models and the saturated models, main models: $B_{\text {movement }}=$ $0.42,95 \% \mathrm{CI}[-0.47,-0.38], p<.001$, and $B_{\text {visitation }}=-0.35,95 \% \mathrm{CI}[-0.46,-0.25], p<.001$, and saturated models: $B_{\text {movement }}=-0.51,95 \% \mathrm{CI}[-0.56,-0.45], p<.001$, and $B_{\text {visitation }}=-0.44,95 \%$ CI[-0.57, -0.32], $p<.001$ (see the highlighted rows in the main model and saturated model in Tables S2 and S3 for the interactions between gender and time). These negative interactions indicate that the link between counties' male percentage and reduced social distancing 
increased between March 9 and May 29, 2020 (see Figures 2a and 2b). Crucially, these

interactions were observed while adjusting for numerous covariates (see above) and for the interaction terms between these covariates and linear time (see Tables S2 and S3).

\section{Figure 2a}

U.S. counties' average social distancing (percent reduction in general movement) as a function of time and gender distribution (More Males vs. More Females; median-split for the purpose of this figure). Dashed lines depict the daily average across counties. Dark lines represent the daily average across counties smoothed with geom_smooth in ggplot (default is loess smooth for $n<$ 1,000). Estimates were composed from raw scores.

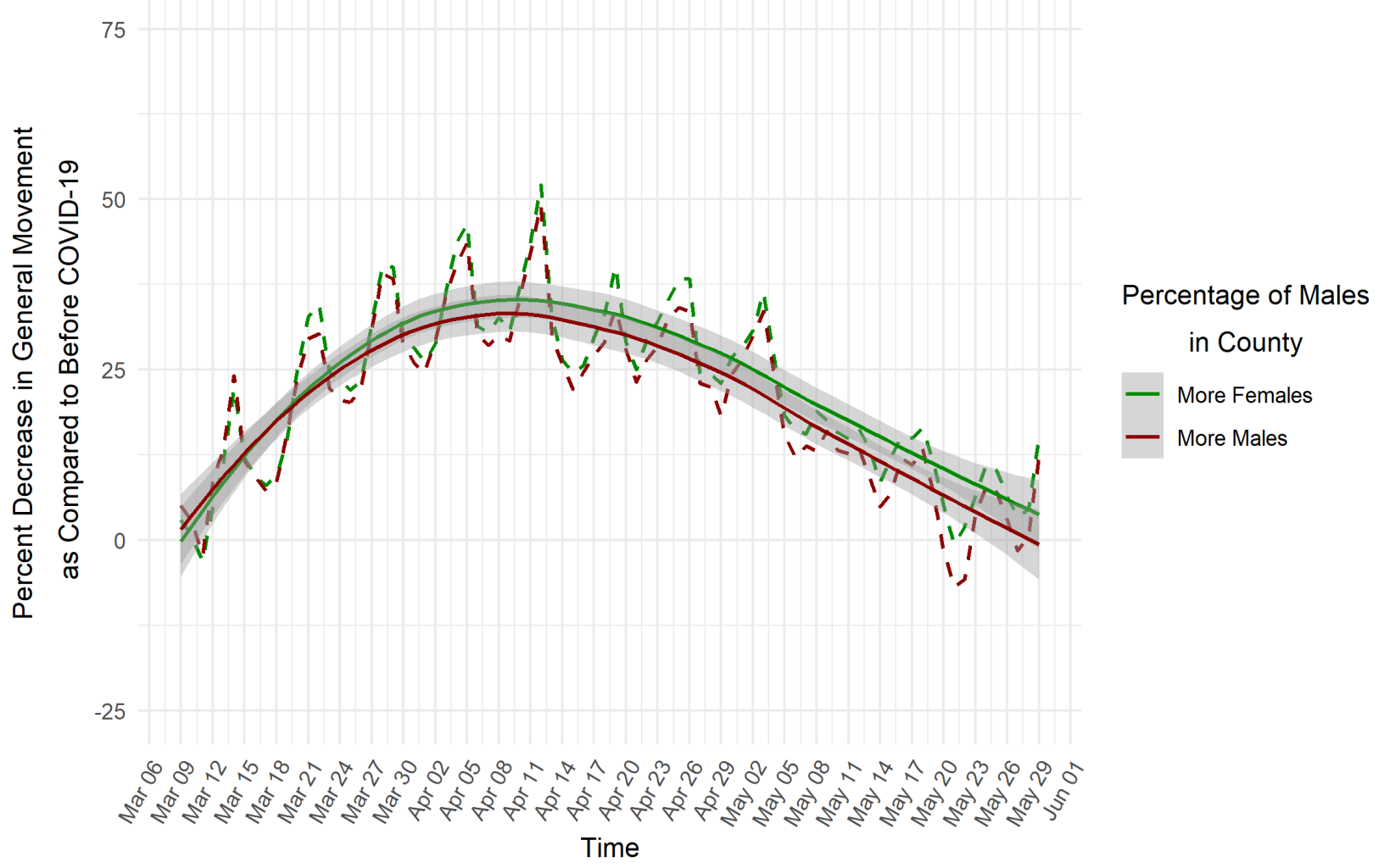

Note. The analysis controlled for general movement pre-Covid-19 per county (movement before March 9, 2020). 


\section{Figure 2b}

U.S. counties' average social distancing (percent reduction in visiting non-essential retail) as a function of time and gender distribution (More Males vs. More Females; median-split for the purpose of this figure). Dashed lines depict the daily average across counties. Dark lines represent the daily average across counties smoothed with geom_smooth in ggplot (default is loess smooth for $n<1,000)$. Estimates were composed from raw scores.

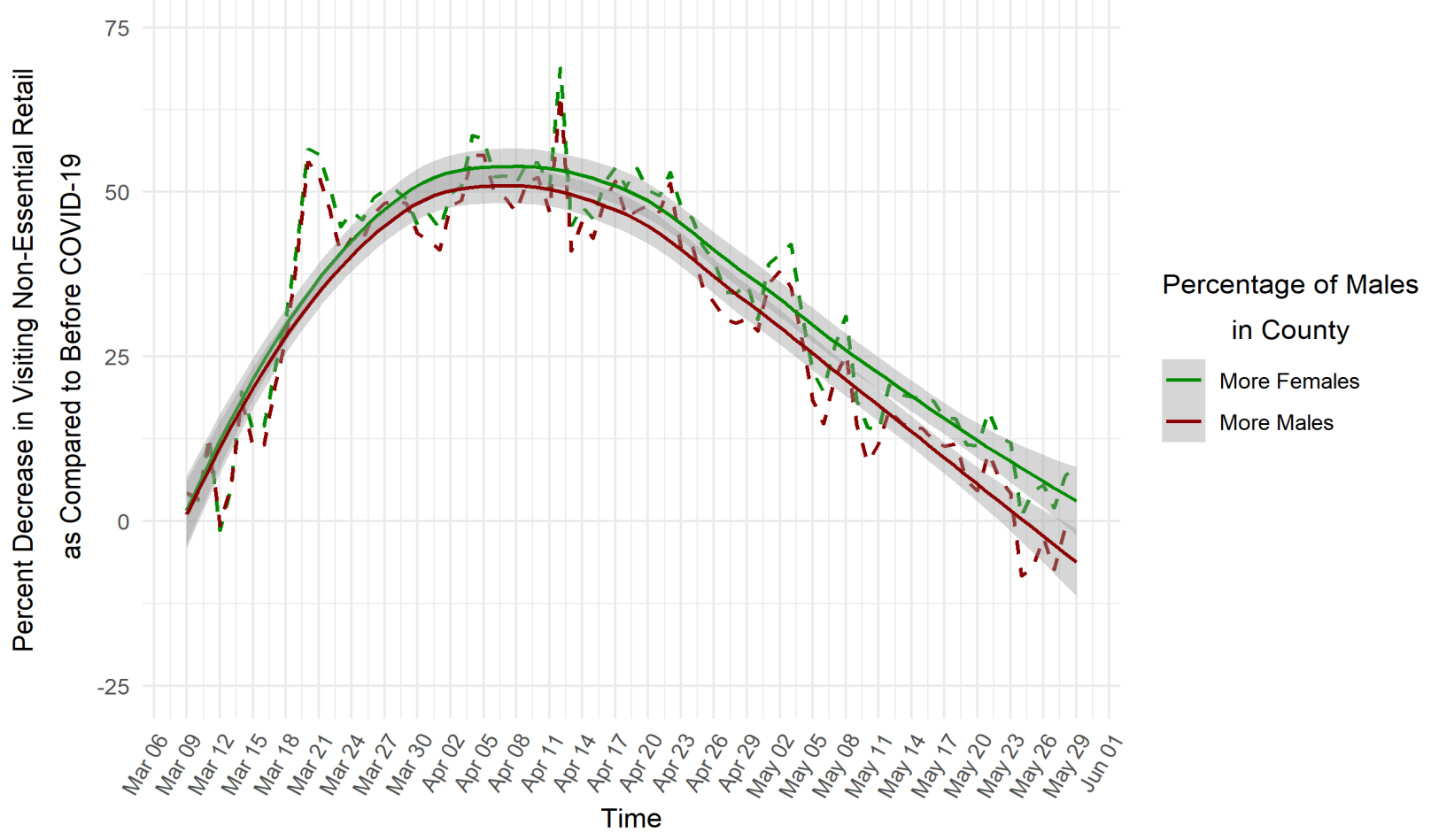

Note. The analysis controlled for visiting non-essential retail pre-Covid-19 per county (visitation before March 9, 2020).

Potentially, though, our findings are driven by men and women holding jobs that differ in terms of being deemed essential during Covid-19 or not. Arguing against this possibility, adding counties' percent of employment in various types of professions to the saturated model (see above) did not account for our findings; the results remained when controlling for counties' percent of workers in agriculture (which also includes forestry, fishing, and hunting), mining (which also includes quarrying, oil, and gas extraction), utilities, construction, manufacturing, wholesale trade, retail trade, transportation and warehousing, information, 
finance and insurance, real estate, professional services (also include scientific and technical services), management of companies and enterprises, administrative, educational services, health care and social assistance, arts (including entertainment and recreation), and finally, accommodation and food services; interaction terms between gender distribution and linear time predicting social distancing: $B_{\text {movement }}=-0.69,95 \% \mathrm{CI}[-0.78,-0.60], p<.001$, and $B_{\text {visitation }}=-1.06,95 \%$ CI[-1.22, -0.90], $p<.001$ (see Table S5; only 1983 and 1744 counties were included in these analyses, respectively, due to missing data in percent employment types).

Additionally, we examined whether the observed interactions decrease in size when adding political orientation to the main and saturated models (in terms of voting gap in the 2016 election). The links between counties' gender distribution and social distancing over time did not substantially decrease, however, when accounting for counties' percentage of votes for Donald Trump over Hillary Clinton in the main models: $B_{\text {movement }}=-0.53,95 \% \mathrm{CI}[-0.58,-0.48], p<.001$, and $B_{\text {visitation }}=-0.31,95 \% \mathrm{CI}[-0.41,-0.20], p<.001$. And, while including political orientation decreased the interaction between gender and time in terms of visiting non-essential retail, $B_{\text {visitation }}=-0.18,95 \% \mathrm{CI}[-0.30,-0.05], p=.006$, it did not do so in terms of general movement, $B_{\text {movement }}=-0.52,95 \%$ CI[-0.57, -0.46$], p<.001$ (Tables S6 and S7).

Potentially, the observed findings are driven by gender differences within households in terms of behavior during the pandemic (e.g., men shopping for groceries). To test this possibility, we examined whether the observed links between gender distribution, time, and social distancing were moderated by counties' total number of family households (weighted by county population) and total number of non-family households (single people) where the householder lives alone (i.e., weighted by county population). We did not find household type to impact our findings; consistent three-way interactions between the two types of social distancing, the two household 
variables and linear time, were not observed. And, for the interactions that were significant, we found that gender differences were actually driven by counties with a higher proportion of single occupants; namely, single women social distancing more than single men rather than family women social distancing more than family men (see Tables S8 and S9; see Figures S3-S6).

\section{Discussion}

In three studies, we observed gender differences in terms of COVID-19 preventative practices. In Study 1, we found that women are more likely to report engaging in social distancing and hygiene practices, as well as to listen to data-driven and internal sources when making social distancing decisions. Due to the potential limitations of self-report survey measures (gender differences may occur more in reporting than in actual behavior), in two other studies, we employed observational and behavioral methodologies to investigate the same question. Specifically, Study 2 extended these findings to actual preventive behavior. We observed that a greater percentage of women than men wear masks in public in three different locations of the Northeast U.S. (New Haven, CT, New York, NY, and New Brunswick, NJ). Finally, Study 3 extended these results to the group level. We examined whether U.S. Counties' gender distribution predicted the degree of social distancing behavior in those counties as assessed via the movement of $\sim 15$ million GPS smart-phone coordinates per day across the United States between March 9 and May 29, 2020. Our analyses revealed that U.S. Counties with more male constituents exhibited decreased social distancing in terms of a smaller reduction in general movement and visiting non-essential retail as COVID-19 progressed.

Exploratory analyses in Study 1 suggested that political ideology may be one factor underlying the gender differences observed in preventive health measures. Consistent with this finding, other research has recently documented that political conservatives engage in less social 
distancing, feel more in control over their own actions regarding the prevention of COVID-19, and feel less responsible for the prevention of the spread of the virus. ${ }^{27,} 28,29$ Although political ideology only partially accounted for the gender differences observed in Study 1 (at the individual level) and did not account for the link between counties' gender distribution and social distancing in Study 3 (at the group-level), future research would benefit from a systematic investigation of the exact role ideology and other ideology-relevant constructs (e.g., masculinity and endorsement of traditional gender roles) play in the observed pattern of results.

\section{Limitations}

There are several limitations of the present study. First, the observed gender differences in social distancing may be explained by structural factors (e.g., employment conditions, family compositions) rather than a personal motivation to maintain preventive health practices. In Study 1, we accounted for one such factor by demonstrating that number of on-site work-days at the time of the study did not account for or interact with the observed gender differences. And, in Study 3, potentially gendered behavior in families (e.g., shopping versus childcare) did not appear to account for the observed results—we found that counties' number of single vs. family households did not moderate our findings. Further, one might argue that a higher tendency to embrace traditional views that align more with conservative ideology can explain the observed gender differences; however, we found our results even when controlling for political orientation in Studies 1 and 3. And, political orientation generally, did not interact with gender to predict social distancing in Study 1. Finally, controlling for factors related to SES (annual income, economic inequality, education, employment, profession type) at the county level in Study 3 did not change our results. Nonetheless, all these county-level factors were interpreted via prepandemic data (e.g., these data did not take into account the shifts in SES as a result of the 
pandemic), and therefore should be interpreted with caution. Future research should investigate the role of behaviors within households and other structural factors that could influence how gender contributes to social distancing decisions and practices as the COVID-19 pandemic was unfolding.

Second, the behavioral observation in Study 2 was constrained to three locations in the North East U.S. where the authors of this study were located during stay-at-home orders. Given the circumstances surrounding COVID-19, we were unable to collect data in locations outside of where we were self-quarantining. Despite these locations varying in terms of annual household income, population of the household, age, and ethnicity, one should be cautious in terms of generalizing our findings to the entire U.S. population. For instance, all three locations were part of Blue states in the U.S. (i.e., voted Clinton over Trump in 2016 elections, both at the state and county level). Although Study 3, which examined millions of data points across the entire United States (including conservative counties), should largely remedy these concerns, future research should still test whether the observed gender differences in mask-wearing extend to other locations and demographics.

Third, the size of the link between counties' gender distribution and social distancing in Study 3 was not particularly large. That is, including additional covariates in the conducted models would likely at some point eliminate the observed effects of counties' gender distribution on social distancing. We note, though, that this would not be particularly surprising given that these covariates would likely be picking up on the psychological variables underlying why maleness is linked to reduced social distancing in the first place (e.g., masculinity threat, risktaking). 
Finally, the present studies do not discount the potential role of biological factors in gender differences in the severity of COVID-19 cases and mortality (e.g., greater prevalence of relevant health problems such as hypertension and cardiovascular diseases among men than women). That is, the current findings are more relevant in terms of understanding potential gender differences in the potential of spreading COVID-19 (due to differences in engaging in preventive health practices) than gender differences in the severity of the cases and mortality rates.

\section{Policy Implications}

Collectively, our results suggest that men may be at higher risk of catching and spreading Coronavirus because of failing to engage in preventative practices. As such, alerting men in particular to the protective power of social distancing, mask-wearing, and hygiene may be helpful in reducing the spread of the virus. Ways to fine-tune preventive health policies towards men may be to target men's potential illusions of invulnerability (that are supported by traditional views of masculinity), ${ }^{20,30}$ and reminding men of their responsibilities towards others as well as themselves during this critical period. ${ }^{8,}{ }^{31}$ Finally, such interventions may be particularly effective in attenuating the harmful consequences of COVID-19 given the higher hospitalization and fatality rates among men so far in the pandemic. ${ }^{32,33,34}$ 


\section{References}

1. Centers for Disease Control and Prevention. (2020, April, 11). How to protect yourself \& others. https://www.cdc.gov/coronavirus/2019-ncov/prevent-getting-sick/prevention.html https://www.cdc.gov/coronavirus/2019-ncov/prevent-getting-sick/prevention.html

2. Tabri, N., Hollingshead, S., \& Wohl, M. J. A. (2020, March 31). Framing COVID-19 as an existential threat predicts anxious arousal and prejudice towards Chinese people. https://doi.org/10.31234/osf.io/mpbtr

3. Aleem, Z. (2020, March 15). A new poll shows a startling partisan divide on the dangers of the coronavirus. Vox. https://www.vox.com/2020/3/15/21180506/coronavirus-poll-democratsrepublicans-trump

4. Bruce, G., Nguyen, H. Ballard, J., \& Sanders, L. (2020, March 27). COVID-19 report: How the virus is affecting everything, from politics to brands. YouGov. https://today.yougov.com/topics/science/articles-reports/2020/03/12/covid-19-report-how-virusaffecting-world-politics

5. Dusenbury, W. (2020, March, 16). Some of Florida's top beaches closed to prevent coronavirus, but not everybody got the message. Sun Sentinel. https://www.sunsentinel.com/coronavirus/fl-ne-clearwater-beach-coronavirus-20200316pag4de6onnauffkaykbfz61654-story.html 6. Rahman, K. (2020, March, 15). St. Patrick's day revelers in Chicago and Louisiana flout coronavirus warnings to stay at home. Newsweek. https://www.newsweek.com/st-patricks-floutcoronavirus-warnings-fill-bars-1492376

7. Waldron, I., \& Johnston, S. (1976). Why do women live longer than men? Journal of Human Stress, 2(2), 19-30. 
8. Umberson, D. (1992). Gender, marital status and the social control of health behavior. Social Science \& Medicine, 34(8), 907-917.

9. Gilligan, C. (1982). In a different voice: Psychological theory and women's development. Cambridge, MA: Harvard University Press.

10. Gilligan, C., \& Wiggins, G. (1988). The origins of morality in early childhood relationships. In C. Gilligan, J. V. Ward, \& J. M. Taylor (Eds.), Mapping the moral domain (pp. 111-138). Cambridge, MA: Harvard University Press.

11. Karniol, R., Grosz, E., \& Schorr, I. (2003). Caring, gender role orientation, and volunteering. Sex Roles, 49(1-2), 11-19.

12. Cheng, Y., Decety, J., Lin, C. P., Hsieh, J. C., Hung, D., and Lee, P. L. (2007). Sex differences in the spinal excitability during observation of bipedal locomotion. Neuroreport, 18 , $887-890$.

13. Marzoli, D., Palumbo, R., Domenico, A. D., Penolazzi, B., and Garganese, P. (2011). The relation between self-reported empathy and motor identification with imagined agents. PLoS One, 6:e14595.

14. Hoffman, M. L. (1977). Sex differences in empathy and related behaviors. Psychological Bulletin, 84, 712-722.

15. Byrnes, J. P., Miller, D. C., \& Schafer, W. D. (1999). Gender differences in risk taking: A meta-analysis. Psychological Bulletin, 125(3), 367-383.

16. Liang, W., Shediac-Rizkallah, M. C., Celentano, D. D., \& Rohde, C. (1999). A populationbased study of age and gender differences in patterns of health-related behaviors. American Journal of Preventive Medicine, 17(1), 8-17. 
17. Gollwitzer, Martel, Marshall, Höhs, \& Bargh, 2020. (preprint). Connecting self-reported social distancing to real-world behavior at the individual and U.S. state level.

18. Reynolds, W. M. (1982). Development of reliable and valid short forms of the MarloweCrowne Social Desirability Scale. Journal of Clinical Psychology, 38(1), 119-125.

19. Kray, L. J., Howland, L., Russell, A. G., \& Jackman, L. M. (2017). The effects of implicit gender role theories on gender system justification: Fixed beliefs strengthen masculinity to preserve the status quo. Journal of Personality and Social Psychology, 112(1), 98-115.

20. Courtenay, W. H. (2000). Constructions of masculinity and their influence on men's wellbeing: a theory of gender and health. Social Science \& Medicine, 50(10), 1385-1401.

21. Oettingen, G., \& Seligman, M. E. (1990). Pessimism and behavioural signs of depression in East versus West Berlin. European Journal of Social Psychology, 20(3), 207-220.

22. Saarni, C. (1984). An observational study of children's attempts to monitor their expressive behavior. Child Development, 1504-1513.

23. Skitka, L. (February, 2019). Presidential Plenary: Behavior Is Worth 1,000 Words. Society of Personality and Social Psychology. Portland, OR.

24. Talhelm, T., Zhang, X., \& Oishi, S. (2018). Moving chairs in Starbucks: Observational studies find rice-wheat cultural differences in daily life in China. Science Advances, 4(4), eaap8469.

25. Cialdini, R. (February, 2019). Which Behavior Speaks Loudest? Society of Personality and Social Psychology. Portland, OR.

26. Unacast. (2020). Social Distancing Scoreboard. https://www.unacast.com/covid19/socialdistancing-scoreboard. 
27. Everett, J. A. C., Colombatto, C., Chituc, V., Brady, W. J., \& Crockett, M. (2020, March 20). The effectiveness of moral messages on public health behavioral intentions during the COVID19 pandemic. https://doi.org/10.31234/osf.io/9yqs8

28. Rothgerber, H., Wilson, T., Whaley, D., Rosenfeld, D. L., Humphrey, M., Moore, A. L., \& Bihl, A. (2020, April 22). Politicizing the COVID-19 Pandemic: Ideological Differences in Adherence to Social Distancing. https://doi.org/10.31234/osf.io/k23cv

29. Gollwitzer, A., Martel, C., Brady, W. J., Pärnamets, P., Freedman, I., Knowles, E., \& Van Bavel, J. J. (2020, May 24). Partisan Differences in Physical Distancing Predict Infections and Mortality During the Coronavirus Pandemic. https://doi.org/10.31234/osf.io/t3yxa

30. Mahalik, J. R., Burns, S. M., \& Syzdek, M. (2007). Masculinity and perceived normative health behaviors as predictors of men's health behaviors. Social Science \& Medicine, 64(11), 2201-2209.

31. White, R. (2002). Social and political aspects of men's health. Health:, 6(3), 267-285.

32. Grasselli, G., Zangrillo, A. \& Zanella, A, et al. (2020). Baseline characteristics and outcomes of 1591 patients infected with SARS-CoV-2 admitted to ICUs of the Lombardy region, Italy. JAMA, 6. https://jamanetwork.com/journals/jama/fullarticle/2764365.

33. Centers for Disease Control and Prevention. (2020). Hospitalization Rates and Characteristics of Patients Hospitalized with Laboratory-Confirmed Coronavirus Disease 2019 - COVID-NET, 14 States, March 1-30, 2020. (Volume. 69).

https://www.cdc.gov/mmwr/volumes/69/wr/pdfs/mm6915e3-H.pdf.

34. China CDC Weekly. (2020). The Epidemiological Characteristics of an Outbreak of 2019 Novel Coronavirus Diseases (COVID-19),-China 2020, 2(8), 113-122. 